\title{
First Demonstration of Phase Contrast Scanning Transmission Electron Microscopy
}

Hiroki Minoda $^{1^{*}}$, Takayuki Tamai ${ }^{1}$, Hirofumi Iijima ${ }^{2}$, Fumio Hosokawa ${ }^{2}$, Yukihito Kondo ${ }^{2}$

${ }^{1}$ Department of Applied Physics, Tokyo University of Agriculture and Technology, 2-24-16 Naka-cho, Koganei, Tokyo 184-8588, Japan,

${ }^{2}$ JEOL Ltd, 3-1-2 Musashino, Akishima, Tokyo 196-8558, Japan

Biological molecules are composed of light elements and can be considered as phase objects for the electron waves. Therefore, images of their unstained specimens show low contrast in transmission electron microscopy (TEM). Phase contrast TEM (P-TEM) is one of the powerful techniques to enhance image contrast of the phase objects [1]. In P-TEM, the phase plate (PP) is placed at a back focal plane (BFP) of the objective lens (OL), and its role is giving a phase shift on a portion of transmitted electron waves, which are scattered or unscattered depending on the type of the PP.

So far, developed PPs are mainly thin film and electrostatic types. The thin film PP is placed on a BFP of the PL and gives a phase shift of $\pi / 2$ to scattered waves by means of the mean inner potential of the PP material. The electrostatic PP consists of three electrodes to modify the phase of the central beam in the diffraction pattern by the electric field. Each one has a hole for the central beam to passing thorough. The three electrodes are placed adequately and a central electrode is connected to a voltage source and the other two are connected to the ground. This configuration forms an electrostatic potential to modify a phase of the unscattered beam passing through the hole of the electrodes In both types, electron waves having a phase shift interfere with electron waves without phase shift. Accordingly, phase shift by the specimen gives image contrast.

Since the PP is placed on the BFP of the OL, there is a limitation in the OL used for the P-TEM. Relatively wide gap OL is needed for P-TEM and a high resolution instrument cannot be used for the $\mathrm{P}$ TEM. Electrostatic charging of the PP is also the important problems in the P-TEM. The size of the central beam in the diffraction pattern is very small and its current density is very high. Electrostatic charging is easy to occur on the way when the PP is placed to an appropriate position because high current density electron beam scans the PP. To overcome this charging problem is one of the important tasks to expand the P-TEM.

Applying the principle of reciprocity to scanning transmission electron microscopy (STEM), the STEM imaging optics is equivalent to the imaging optics in the conventional TEM. Therefore, a phase contrast scanning transmission electron microscopy (P-STEM) can be used to enhance phase contrast of the phase objects. In the P-STEM, a PP can be set on the condenser lens aperture (CLA) plane that is optically equivalent to a front focal plane (FFP) of an OL. Figure 1 compares the imaging optics of the P-TEM and P-STEM. There are larger spaces around the CLA plane than that round the BFP of the OL. The electron beam is not focused on the CLA plane and the current density of the electrons illuminated to the PP on the CLA plane is much lower than that on the BFP of the OL. A total electric current illuminated to the PP on the CLA plane is higher than that on the BFP of the OL. This seems to cause more serious electrostatic charging problems in the P-STEM rather than P-TEM. However, a current density is experimentally proved to be more serious for the charging problems of the PP than the total current. In addition, we could see the condition of the PP by using the Ronchigram in P-STEM which is one of the advantages of the P-STEM to evaluate the condition of the PP. In this paper, the application of the phase plate microscopy to the STEM mode is demonstrated. 
[1] R. Danev and K. Nagayama, J. Phys. Sci. Jpn. 70 (2001), 696.

[2] This development was supported by SENTAN, JST.

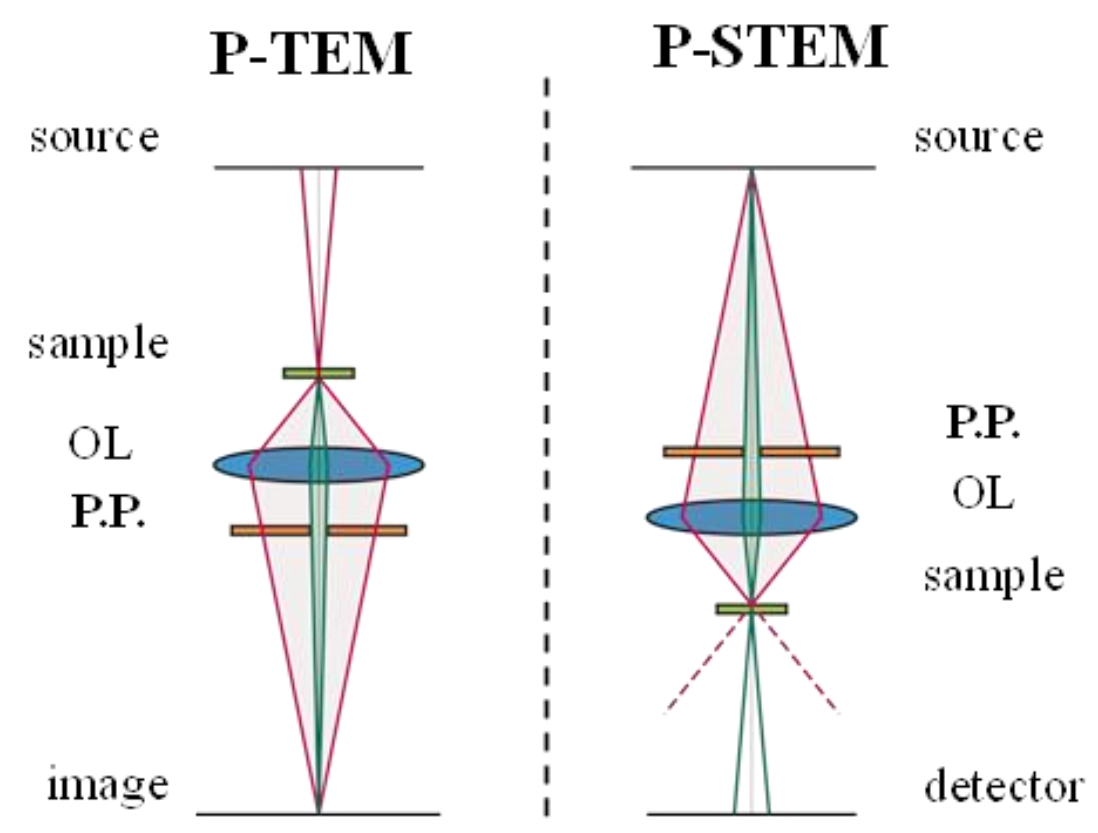

Figure 1. A comparison of the imaging optics of the P-TEM (left) and P-STEM (right). 